\title{
Increasing and Dispersing Strain in Pyrene-Fused Azaacenes
}

\author{
Javier Mateos-Martín, ${ }^{a}$ Marco Carini, ${ }^{a}$ Manuel Melle-Franco ${ }^{* b}$ and Aurelio Mateo-Alonso*ac
}

Received 00th January 20xx, Accepted 00th January 20xx

DOI: $10.1039 / x 0 \times x 00000 x$

\begin{abstract}
A new strategy to obtain distorted pyrene-fused azaacenes is reported. The careful alignment and selection of substituents give rise to highly twisted pyrene-fused azaacenes. A combined global and local theoretical analysis shows how the strain is generated and dispersed across the aromatic backbone. Furthermore, simulation of the observed optoelectronic properties shines light on the structural factors that govern the properties of twisted pyrenefused azaacenes.
\end{abstract}

Strained polycyclic aromatic hydrocarbons ${ }^{1}$ are receiving increasing attention as synthetic targets due to their particular optoelectronic -and in some cases chiroptical- properties, enhanced solubility and curved $\pi$-surfaces that can lead to exotic solid-state packings and to supramolecular recognition of other curved aromatics. Overcrowding has proven to be a very general and efficient approach to introduce strain across a large number of aromatic systems. ${ }^{1}$ Among these overcrowded systems, ribbon-like pyrene-fused twistacenes, ${ }^{1 k}, 2$ which combine a longitudinally-twisted (helical or alternated) aromatic framework with periodic off-linear bisannulations, have emerged not only as challenging compounds from a synthetic perspective, but also as promising materials for $\mathrm{OFET}^{3}$ and OLED 4 applications. Several approaches have been used to induce strain in pyrene-fused acenes, which include phenyl, ${ }^{5}$ ethynylsilyl,1k, 6 cyanide $^{3 b}$ and bisamide ${ }^{7}$ overcrowding. Ethynylsilyl overcrowding has shown to be an effective approach in pyrene-fused azaacenes ${ }^{6}$ and in other nitrogenated systems, ${ }^{8}$ which are generally more difficult to distort because of the shorter $\mathrm{C}=\mathrm{N}$ bonds. Even if twisted pyrene-fused azaacenes have been successfully obtained by this approach, virtually no changes on their optoelectronic properties had been observed in comparison to less strained systems. ${ }^{6}$ This is mostly because of the misalignment between the bulky groups along the aromatic backbone as the result of the offset between the groups sitting on the pyrene and the quinoxaline residues that reduces the strain (Figure 1a).

\footnotetext{
a.POLYMAT, University of the Basque Country UPV/EHU, Avenida de Tolosa 72, E20018, Donostia-San Sebastián, Spain.E-mail: amateo@polymat.eu

b. CICECO - Aveiro Institute of Materials, Department of Chemistry, University of Aveiro, 3810-193, Aveiro, Portugal. E-mail: manuelmelle.research@gmail.com c. Ikerbasque, Basque Foundation for Science, Bilbao, Spain.

+ Electronic Supplementary Information (ESI) available: Experimental details for the synthesis, characterization and calculations.
}

a)

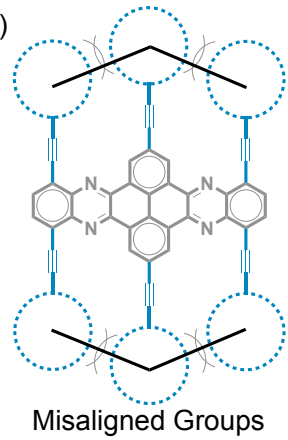

b)

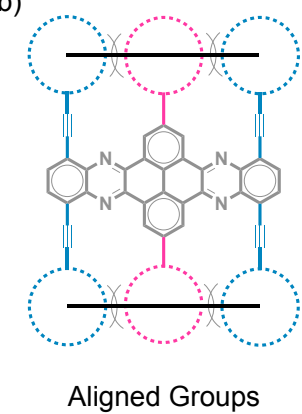

Figure 1 Pyrene-fused azaacenes with a) misaligned and b) aligned groups.

Herein we report a new strategy to obtain twisted pyrenefused azaacenes, which relies on the careful alignment of all the sterically-demanding groups along the aromatic backbone. This alignment has been achieved by eliminating the (acetylene) offset in the pyrene residue (Figure 1 b) by attaching directly the bulky groups on the 2,7 positions of the pyrene residue. The careful alignment of groups results in an enhancement of the steric interactions between them and gives rise to pyrene-fused azaacenes that show larger twist angles than those previously reported and remarkably also show strain-dependent changes on their optoelectronic properties. A theoretical analysis of the molecular structures provides a detailed picture on how the strain is generated and dispersed across the aromatic backbone and on its effects on the optoelectronic properties of twisted pyrene-fused azaacenes.

The synthesis of group-aligned pyrene-fused acenes is achieved by cyclocondensation between pyrene-4,5,9,10tetraone and o-diamine derivatives equipped with bulky groups. 2,7-Di-tert-butylpyrene-4,5,9,10-tetraone (1) was synthesized according to a procedure described on the literature, ${ }^{9}$ while pyrene-4,5,9,10-tetraone with either triisopropylsilyl (TIPS) or triphenylsilyl (TPS) groups on the 2,7 positions had not been previously described and were prepared through a novel synthetic route that involve a novel reductive protection and oxidative deprotection of pyrene quinones, which in turn allows a key lithiation/nucleophilic substitution 
step (Scheme 1a). The starting point is compound $\mathbf{2}$, that can be obtained from pyrene in two steps following a reported procedure. ${ }^{9-10}$ Pyrene 2 was reduced with $\mathrm{Na}_{2} \mathrm{~S}_{2} \mathrm{O}_{4}$ and alkylated in situ with $n$-hexyl bromide to provide the corresponding iodotetraether 3 (63\%). Lithiation of 3 followed by silylation, either with triisopropylsilyl chloride or with triphenylsilyl chloride, yielded 4 (70\%) and 5 (58\%), respectively. Any attempts to perform this lithiation with the diketal-protected $2^{11}$ failed because of its limited solubility in THF. Oxidative ether hydrolysis using $\mathrm{Br}_{2}$ and cerium-ammonium-nitrate (CAN) regenerated the tetraones in the $K$ regions affording pyrenetetraones $6(47 \%)$ and 7 (45\%) as red solids. In addition, crystal suitable for X-Ray diffraction confirmed the structure for 7 (Figure S1).

We then focused on the synthesis of $o$-diamine precursors (Scheme 1b). We selected tris(trimethylsilyl)silyl (TTMS) groups, also known as supersilyl groups, because of their large volume and rigidity, and high solubilizing power. TTMS-acetylene 8 (95\%) was synthesized from commercially available TTMS chloride and ethynyl magnesium bromide. Reaction of $\mathbf{8}$ with dibromobenzothiadiazole 9 under Sonogashira conditions afforded benzothiadiazole 10 (74\%). Reduction of benzothiadiazole ring with $\mathrm{LiAlH}_{4}$ produced the desired 0 diamine 11 (35\%).

Cyclocondensations of the 2,7-substituted tetraketopyrenes 1, 6 and 7, independently, with diamine $\mathbf{1 1}$ (Scheme 3) were performed in acetic acid at $40{ }^{\circ} \mathrm{C}$ yielding the desired dibenzotetraazahexacenes 12 (83\%), 13 (40\%) and 14 (47\%).

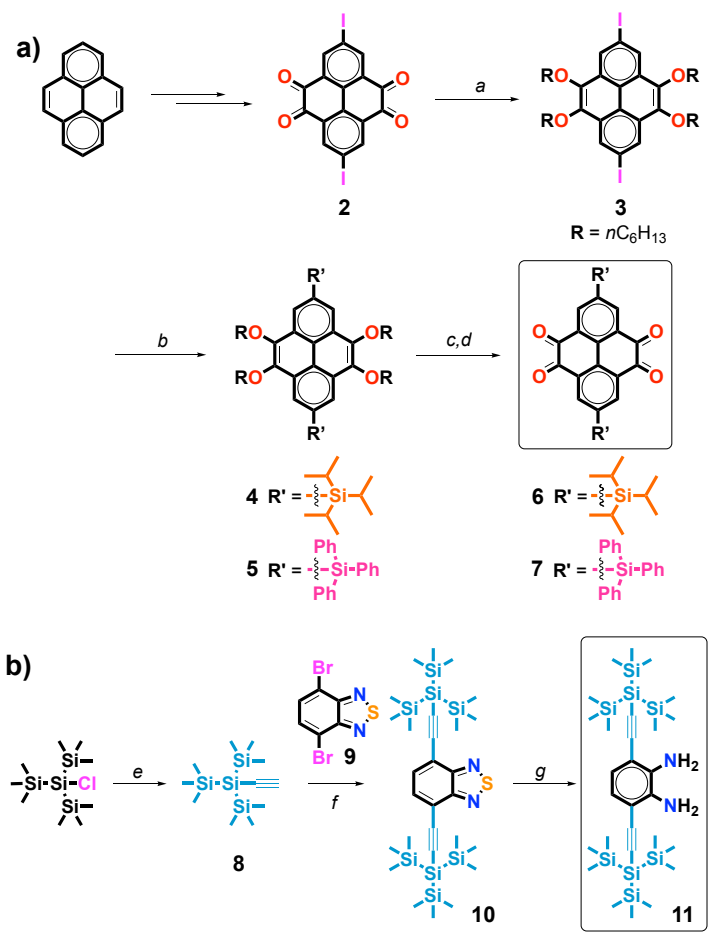

Scheme 1 Synthesis of 6, 7 and 11. Reagents and conditions: a) TBAB, $\mathrm{Na}_{2} \mathrm{~S}_{2} \mathrm{O}_{4}$, DMF/ $\mathrm{H}_{2} \mathrm{O} 3: 2, \mathrm{~K}_{2} \mathrm{CO}_{3}, n-\mathrm{C}_{6} \mathrm{H}_{13} \mathrm{Br}, 100{ }^{\circ} \mathrm{C}, 4 \mathrm{~h}, 63 \%$; b) n-BuLi (1.6M), THF, iPr $\mathrm{PriCl}_{3}$ or $\mathrm{Ph}_{3} \mathrm{SiCl},-80{ }^{\circ} \mathrm{C}$ to r.t., overnight, $70 \%$ for $4,58 \%$ for $\left.5 ; c\right) \mathrm{Br}_{2}, \mathrm{Na}_{2} \mathrm{CO}_{3}, \mathrm{CHCl}_{3}$, $0{ }^{\circ} \mathrm{C}$ to r.t., $10^{\prime}$; d) $\mathrm{CAN}, \mathrm{CHCl}_{3} / \mathrm{MeCN} 1: 5,70^{\circ} \mathrm{C}$, overnight, $47 \%$ for $6,45 \%$ for 7. e) ethynyl magnesium bromide (1.0 M), THF, $0{ }^{\circ} \mathrm{C}$ to r.t., overnight, $95 \%$; f) $\mathrm{PdCl}_{2}\left(\mathrm{PPh}_{3}\right)_{2}, \mathrm{Et}_{3} \mathrm{~N} / \mathrm{THF}$, Cul, r.t., $74 \%$; g) $\mathrm{LiAlH}_{4}, \mathrm{Et}_{2} \mathrm{O},-15^{\circ} \mathrm{C}, 35 \%$.

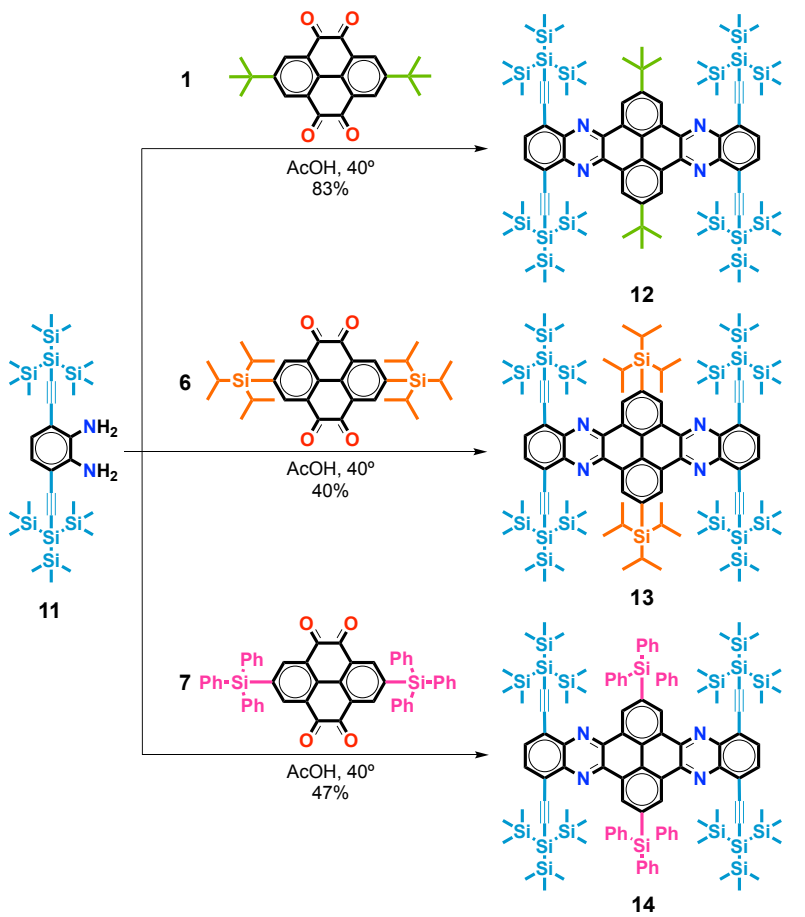

Scheme 2 Synthesis of dibenzotetraazahexacenes 12, 13 and 14.

Crystals suitable for X-Ray diffraction were obtained by vapor diffusion of ethanol into a solution of the corresponding dibenzotetraazahexacene in toluene. Crystal structures of 12-14 (Figure 2) show an evident steric interaction between TTMS and the different substituents sitting on the pyrene residue as the result of the alignment of the groups along the aromatic backbone. This interaction is evidenced by the bent structure of the acetylenes and the alternated twists along the dibenzotetraazahexacene backbone in which the quinoxaline residues are practically on the same plane and the pyrene residue is out of plane. As the size of the substituents increases, the twist angle between the pyrene and the quinoxaline moieties increases, which is reflected by the end-to-center dihedral angle $\left(\theta_{\mathrm{ABCD}}\right)$ and the quinoxaline-pyrene angle $\left(\theta_{\mathrm{EFGH}}\right)$ values (the largest dihedral angles are reported). For instance, as the size of the groups sitting on the pyrene residues increases ( $t B u<$ TIPS $<$ TPS $)$ the $\theta_{A B C D}\left(3^{\circ}, 17^{\circ}\right.$ and $21^{\circ}$, respectively) and $\theta_{\mathrm{EFGH}}\left(10^{\circ}, 21^{\circ}\right.$ and $31^{\circ}$, respectively) values increase. The largest $\theta_{\mathrm{EFGH}}$ value for 14 is $7^{\circ}$ larger than that previously reported for a pyrene-fused azaacene with misaligned TIPS substituents. ${ }^{6}$ The average deformation of the acetylenes increases from $\mathbf{1 2}$ to 13 $\left(7.4^{\circ}, 8.5^{\circ}\right.$ and $\left.8.4^{\circ}\right)$, while in the case of 14 , it remains practically the same as for 13 . The bond lengths observed on the crystal structures are in good agreement with the geometries calculated for 12-14 at the B3LYP-6-31G(d,p) level (Figure S2), with calculated nucleus-independent chemical shifts (NICS) (Figures S3 and S4) and also with Clar rules that also predict a naphthalene group in the quinoxaline residues and two aromatic sextets in the off-linear rings of the pyrene residue. To shine light on the influence of strain on the structure, we computed both global and bond strain energies. The total strain energies for 12, 13 and 14 (4.0, 8.2 and $11.2 \mathrm{kcal} / \mathrm{mol}$, 

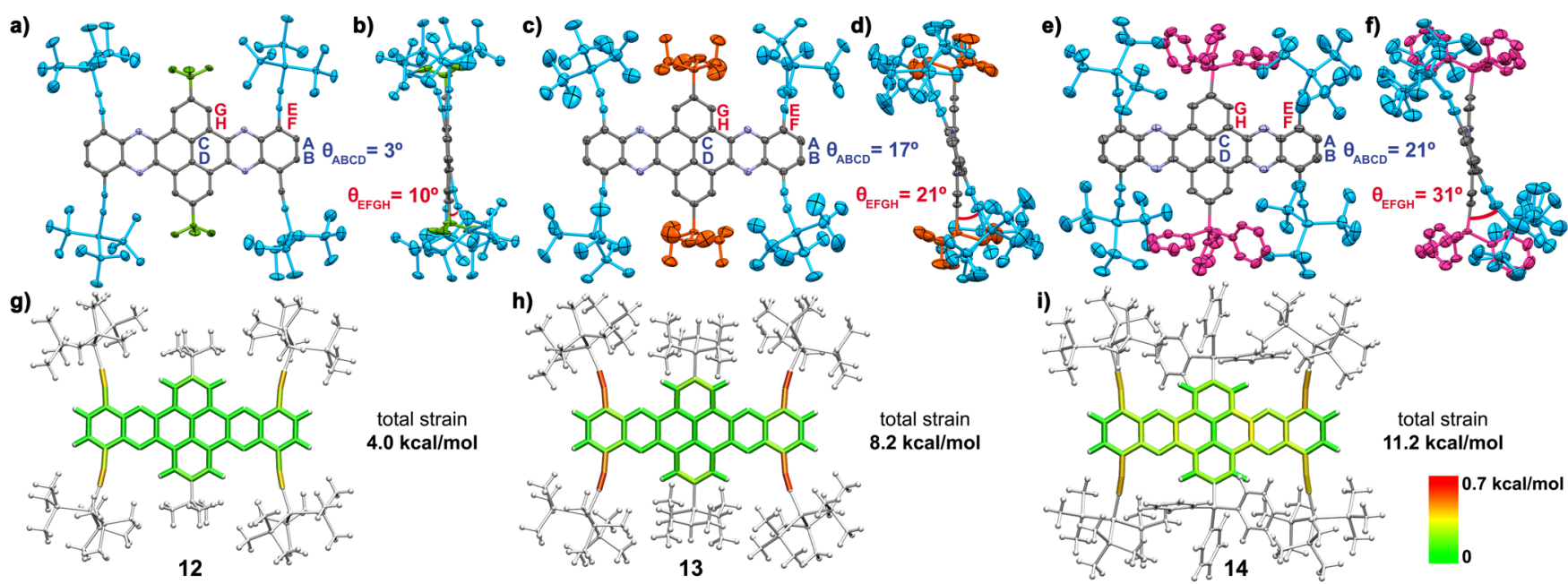

Figure 2 Frontal view $(a, c, e)$ and side view (b, d, f) of X-ray structures (ellipsoids at 50\% probability level) where hydrogen atoms were omitted for clarity. Strain bond maps and total strain energies computed at B3LYP-6-31G(d,p) level (g, h, and i) of 12, 13 and 14.

respectively) computed at the B3LYP-6-31G(d,p) level also reflect how the increasing size of the substituents in the pyrene residue increases substantially the strain (Figure $2 \mathrm{~g}-\mathrm{i}$ ). The local strain was estimated with an in-house modification of the software StrainViz developed by Jasti and co-workers. ${ }^{12}$ The strain bond maps show how the steric interactions between the aligned TTMS and $t \mathrm{Bu}$ substituents in $\mathbf{1 2}$ induce strain mostly over the acetylenes, meanwhile virtually no strain is observed on the aromatic core. In 13, when the $t$ Bu groups are exchanged for larger TIPS groups, a substantial increase of local strain is induced on the acetylene residues and again almost no strain is observed on the aromatic core. Lastly, in 14, although the larger TPS groups induces a substantial strain on the acetylenes, this is smaller in comparison to $\mathbf{1 3}$. However, in this case, the strain over the dibenzotetraazahexacene core increases substantially. Considering the strain distribution and the calculated total strain, we can conclude that the strain accumulates preferentially on the acetylene residues. But as the total strain increases as the result of the larger substituents, the acetylenes cannot accommodate all the strain, which is released over the aromatic core.

The optoelectronic and redox properties were investigated by steady-state absorption and fluorescence spectroscopy and cyclic voltammetry. The electronic absorption spectra of 12-14 (Figure 3a, Table S1) show the typical $\alpha, \rho$ and $\beta$ bands observed in pyrene-fused acene systems. ${ }^{13}$ The $\alpha$ band appears as a shoulder in all cases because of the overlap with the $\rho$ band, but in the case of $\mathbf{1 4}$ is bathochromically-shifted by at least $8 \mathrm{~nm}$. These differences are clearly discerned in the fluorescence spectra $\left(\lambda_{\text {exc }}=453 \mathrm{~nm}\right.$, Figure $3 \mathrm{~b}$, Table S1) that show a similar unresolved florescence band that appears at increasingly longer wavelengths from 12 to $\mathbf{1 4}$ and similar quantum yields $\left(\lambda_{\mathrm{em}}=\right.$ 520,524 , and $538 \mathrm{~nm}$; $\Phi=0.18,0.17$ and 0.20; respectively), which in the case of $\mathbf{1 4}$ is bathochromically-shifted by as much as $18 \mathrm{~nm}$. The cyclic voltammograms of 12-14 (Figure S5, Table S1) showed two one electron reversible reduction processes with almost identical redox potentials $\left(E_{1}=-1.61,-1.67\right.$ and $-1.64 \mathrm{~V}$ and $E_{2}=-1.72,-1.78$ and $-1.75 \mathrm{~V}$ respectively). No oxidation processes were observed within the solventsupported electrolyte window. The HOMO-LUMO gaps (2.53, 2.52 and $2.48 \mathrm{eV}$, respectively for 12,13 and 14 ) estimated from the onset of the lowest energy absorption band and the electrochemical LUMO energies or electron affinities (-3.26, 3.17 and $-3.21 \mathrm{eV}$, respectively for 12,13 and 14 ) estimated from the onset of the first reduction waves are very similar in all cases and in agreement with calculations (Table S2).
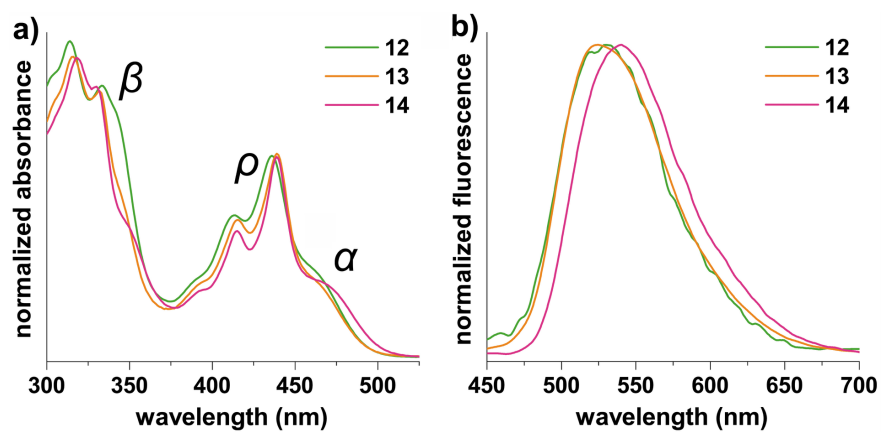

Figure 3 (a) Normalized absorption and (b) fluorescence of 12-14 in $\mathrm{CH}_{2} \mathrm{Cl}_{2}$.

Theoretical calculations were carried out to shine light on the different optoelectronic properties observed for 12-14. The calculated frontier orbitals show that the HOMO-1/HOMO and LUMO/LUMO+1 couples are quasidegenerate in all cases (around -5.7 and $-2.8 \mathrm{eV}$, respectively). However, meanwhile virtually identical electronic densities for the different frontier orbitals are observed for $\mathbf{1 2}$ and $\mathbf{1 3}$ (Figure S6), in the case of 14, clear differences can be observed. For instance, the HOMO-1 and HOMO orbitals show density on both quinoxaline residues of 12 and 13, but in the case of 14, the electronic densities are localized in opposite quinoxaline residues in the HOMO-1 and HOMO, respectively. Also, while in the case of 12 and 13, the LUMO and LUMO+1 densities are almost identical, but in the case of 14 LUMO and the LUMO+1 are exchanged. Furthermore, TD-DFT calculations (Table S3, Figure S7) show that the lowest energy $\alpha$ band in the electronic absorption spectra of $\mathbf{1 2}$ and $\mathbf{1 3}$ corresponds to a HOMO-1 $\rightarrow$ LUMO and HOMO $\rightarrow$ LUMO +1 transitions (the HOMO $\rightarrow$ LUMO and HOMO-1 $\rightarrow$ LUMO+1 are 
dark transitions). However, in the case of $\mathbf{1 4}$ the $\alpha$ band shows, in addition to the contribution of the HOMO-1 $\rightarrow$ LUMO and HOMO $\rightarrow$ LUMO+1 transitions, the contribution of the HOMO $\rightarrow$ LUMO and HOMO-1 $\rightarrow$ LUMO+1 transitions, which altogether account for the differences observed in the experimental absorption and fluorescence spectra.

To understand whether these effects are result of the different substituents or of the twists across the $\pi$-system, the structure and the absorption spectra (TD-B3LYP- $\mathrm{CH}_{2} \mathrm{Cl}_{2}-6$ $31 G(d, p) / B 3 L Y P-6-31 G(d, p)$ level) of a progressively twisted (from $0^{\circ}$ to $60^{\circ}$, in $10^{\circ}$ steps) model dibenzotetraazahexacene, in which the silyl substituents have been replaced by hydrogens, were computed (Figure 4). First, the twisting energy increases monotonically but not linearly with the twist angle. Also, as the twist angle increases, the peak maximum wavelength of the $\alpha$ band is increasingly red-shifted (Figure S8) as observed experimentally. Furthermore, at twist angles $>30^{\circ}$, the LUMO density corresponds to the LUMO+1 density of the flat system, exactly as we observed for $\mathbf{1 4}$ with respect to $\mathbf{1 2}$ and $\mathbf{1 3}$ (Figures 4 and 56). Therefore the differences in the optoelectronic properties observed in $\mathbf{1 4}$ can be attributed to the distortion of the $\pi$-system.

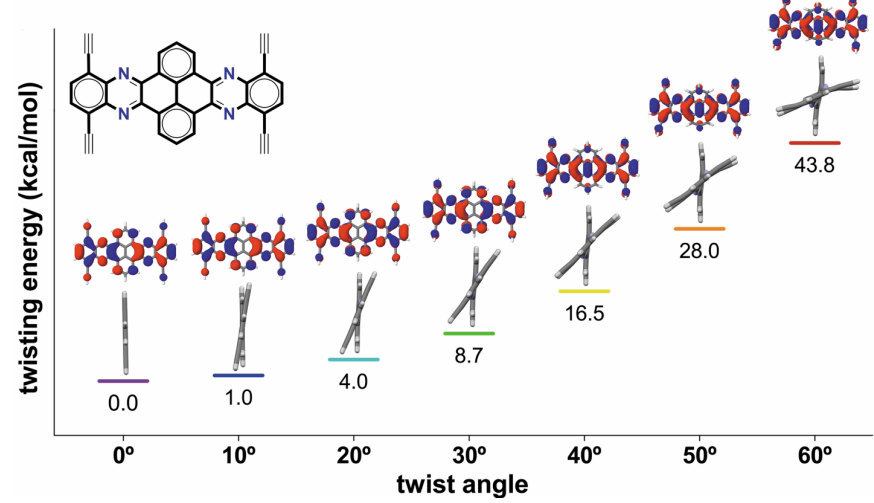

Figure 4 a) Twisting energy as a function of the angle from $0^{\circ}$ flat, $D_{2 h}$ symmetry, to $60^{\circ}$ computed by partial optimizations at the B3LYP-6-31G(d,p) level. Front views (with LUMO density) and side views are shown for each selected angle.

To conclude, we have described here a new approach to increase twist angles in pyrene-fused azaacenes up to $31^{\circ}$ by the careful alignment and selection of sterically demanding groups. Remarkably different electronic absorption and fluorescence properties have been observed as a result of the higher strain. To achieve this, we developed a synthetic methodology that allows introducing silyl groups in the 2,7 positions of pyrene4,5,9,10-tetraone derivatives by a novel reductive protection and oxidative deprotection of pyrene quinones that makes possible a key lithiation/nucleophilic substitution step. In addition, the application of a novel strain local analysis together with the perusal of the electronic structure on three different pyrene-fused azaacenes has provided a comprehensive picture on how the strain is induced by the different substituents and propagates across the aromatic backbone, and also, on the effects of such strain on the optoelectronic properties. Overall, this work opens up new possibilities for the design and synthesis of strained aromatics with increasing complexity and tailored properties.

\section{Conflicts of interest}

There are no conflicts to declare.

\section{Acknowledgements}

This work was carried out with the support of the Basque Science Foundation for Science (Ikerbasque), POLYMAT, Diputación Foral de Guipuzkoa, the University of the Basque Country (Grupo de Investigación GIU17/054), Gobierno de España (Ministerio de Economía y Competitividad CTQ2016-77970-R and FPI Grant BES2017-080115), Gobierno Vasco (PIBA 2019-09 and BERC programme), the Portuguese Foundation for Science and Technology/MCTE through the project IF/00894/2015 and within the scope of the project CICECO-Aveiro Institute of Materials, UIDB/50011/2020 \& UIDP/50011/2020 and acknowledge technical and human support provided by SGIker of UPV/EHU and European funding (ERDF and ESF). This project has received funding from the European Research Council (ERC) under the European Union's Horizon 2020 research and innovation programme (Grant Agreement No. 722951).

\section{Notes and references}

1. (a) M. A. Petrukhina, L. T. Scott and H. W. Kroto, Fragments of Fullerenes and Carbon Nanotubes: Designed Synthesis, Unusual Reactions, and Coordination Chemistry, Wiley, 2011; (b) M. Ball, Y. Zhong, Y. Wu, C. Schenck, F. Ng, M. Steigerwald, S. Xiao and C. Nuckolls, Acc. Chem. Res., 2015, 48, 267-276; (c) Y. Segawa, A. Yagi, K. Matsui and K. Itami, Angew. Chem. Int. Ed. , 2016, 55, 5136-5158; (d) S. H. Pun and Q. Miao, Acc. Chem. Res., 2018, 51, 1630-1642; (e) M. A. Majewski and M. Stępień, Angew. Chem. Int. Ed. , 2019, 58, 86-116; (f) R. A. Pascal, Chem. Rev., 2006, 106, 4809-4819; (g) A. Bedi and O. Gidron, Acc. Chem. Res., 2019, 52, 2482-2490; (h) Y. Shen and C.F. Chen, Chem. Rev., 2012, 112, 1463-1535; (i) M. Gingras, Chem. Soc. Rev., 2013 42, 1051-1095; (j) M. Rickhaus, M. Mayor and M. Juríček, Chem. Soc. Rev., 2016, 45, 1542-1556; (k) A. Mateo-Alonso, Eur. J. Org. Chem., 2017, 2017, 7006-7011.

2. (a) Z. Zhang and Q. Zhang, Mater. Chem. Front., 2020; (b) J. Li, S. Chen, Z. Wang and Q. Zhang, Chem. Rec., 2016, 16, 1518-1530.

3. (a) P.-Y. Gu, Z. Wang, G. Liu, H. Yao, Z. Wang, Y. Li, J. Zhu, S. Li and Q. Zhang, Chem. Mater., 2017, 29, 4172-4175; (b) M. Martinez-Abadia, G. Antonicelli, E. Zuccatti, A Atxabal, M. Melle-Franco, L. E. Hueso and A. Mateo-Alonso, Org. Lett., 2017, 19, 1718-1721

4. D. Cortizo-Lacalle, A. Pertegás, M. Melle-Franco, H. J. Bolink and A. Mateo-Alonso, Org. Chem. Front., 2017, 4, 876-881.

5. (a) J. Li, Y. Zhao, J. Lu, G. Li, J. Zhang, Y. Zhao, X. Sun and Q. Zhang, J. Org. Chem. 2015, 80, 109-113; (b) D. Rodriguez-Lojo, D. Perez, D. Pena and E. Guitian, Chem. Commun., 2015, 51, 5418-5420; (c) W. Chen, X. Li, G. Long, Y. Li, R. Ganguly, M. Zhang, N. Aratani, H. Yamada, M. Liu and Q. Zhang, Angew. Chem., 2018, 130, 13743-13747.

6. S. More, S. Choudhary, A. Higelin, I. Krossing, M. Melle-Franco and A. Mateo-Alonso, Chem. Commun., 2014, 50, 1976-1979.

7. W. Fan, T. Winands, N. L. Doltsinis, Y. Li and Z. Wang, Angew. Chem. Int. Ed., 2017, 56, 15373-15377.

8. (a) D. Cortizo-Lacalle, A. Pertegás, L. Martínez-Sarti, M. Melle-Franco, H. J. Bolink and A. Mateo-Alonso, J. Mater. Chem. C, 2015, 3, 9170-9174; (b) A. B. Marco, D. Cortizo-Lacalle, I. Perez-Miqueo, G. Valenti, A. Boni, J. Plas, K. Strutynski, S. De Feyter, F. Paolucci, M. Montes, A. N. Khlobystov, M. Melle-Franco and A. MateoAlonso, Angew. Chem. Int. Ed., 2017, 56, 6946-6951.

9. J. Hu, D. Zhang and F. W. Harris, J. Org. Chem., 2005, 70, 707-708.

10. J. A. Letizia, S. Cronin, R. P. Ortiz, A. Facchetti, M. A. Ratner and T. J. Marks, Chem. Eur. J., 2010, 16, 1911-1928.

11. S. More, R. Bhosale, S. Choudhary and A. Mateo-Alonso, Org. Lett., 2012, 14, 4170 4173.

12. C. E. Colwell, T. W. Price, T. Stauch and R. Jasti, Chem. Sci., 2020, 11, 3923-3930.

13. (a) D. Cortizo-Lacalle, J. P. Mora-Fuentes, K. Strutynski, A. Saeki, M. Melle-Franco and A. Mateo-Alonso, Angew. Chem. Int. Ed., 2018, 57, 703-708; (b) D. CortizoLacalle, C. Gozalvez, M. Melle-Franco and A. Mateo-Alonso, Nanoscale, 2018, 10, 11297-11301; (c) J. P. Mora-Fuentes, A. Riaño, D. Cortizo-Lacalle, A. Saeki, M. MelleFranco and A. Mateo-Alonso, Angew. Chem. Int. Ed., 2019, 58, 552-556; (d) Z. Wang, P. Gu, G. Liu, H. Yao, Y. Wu, Y. Li, G. Rakesh, J. Zhu, H. Fu and Q. Zhang, Chem. Commun., 2017, 53, 7772-7775. 